\title{
Producción científica española en rehabilitacion y fisioterapia a través de la Web of Science
}

The Spanish scientific production in rehabilitation and physiotherapy through the Web of Science

\author{
Victoria Calvo fuente (1), Gisela Cantos Mateos (2), María Ángeles Zulueta García (3)
}

(1) Facultad de Enfermería y Fisioterapia, Universidad de Alcalá, Alcalá de Henares, España, victoria.calvo@uah.es; (2) Instituto de Políticas y Bienes Públicos, Consejo Superior de Investigaciones Científicas, Madrid, España, gisela.cantos@csic.es (3) Facultad de Documentación, Universidad de Alcalá, Alcalá de Henares, España, zulueta@uah.es

\begin{abstract}
Resumen
Se presentan los resultados del análisis de la producción científica desarrollada en España en las áreas de rehabilitación y fisioterapia, recogida en la Web of Science. Para ello se realiza un estudio descriptivo retrospectivo con los documentos que contiene la base de datos hasta 2011, determinando entre otros indicadores bibliométricos: la tipología documental, la producción por zonas geográficas, sectores institucionales, centros y categorías temáticas. Los resultados se obtienen tras el análisis de 1302 documentos, de los cuales el $75,19 \%$ son artículos científicos. Los sectores institucionales más productivos son los hospitales $(54,8 \%)$ y la universidad $(43,9 \%)$. Las Comunidades autónomas más productivas son Madrid $(28,2 \%)$ y Cataluña $(25,5 \%)$, así como sus diferentes centros hospitalarios y universitarios de referencia. Las categorías predominantes resultan ser neurología clínica y rehabilitación.
\end{abstract}

Palabras clave: Fisioterapia. Rehabilitación. Bibliometría. España.

\section{Introducción}

La Fisioterapia es una disciplina transversal que forma parte de la Rehabilitación, de manera que para contextualizarla podemos abordar su estudio dentro del área de la Rehabilitación. En nuestro país aún es una disciplina relativamente joven, hasta hace unos años definida por su marcado carácter asistencial. Recientemente, la remodelación de los planes de estudio y su adaptación al espacio europeo ha supuesto el detonante para incorporarse plenamente al desarrollo de actividades científicas e investigadoras. La investigación es esencial para definir esta disciplina y constituye el mejor vehículo de comunicación entre investigadores y clínicos (Torres, 2012), lo que favorece la mejora de la calidad asistencial y de la formación académica.

El crecimiento de la producción científica en esta área potencia la realización de estudios bibliométricos, que aportan una visión interesante de la actividad científica, tanto del propio país

\begin{abstract}
The results of a scientific production analysis developed in Spain in the fields of Rehabilitation and Physiotherapy are presented, providing an overview of Spanish research in the fields. In order to this, a retrospective descriptive study was performed with documents covered by the Web of Science until 2011, defining among other bibliometric indicators: document typology, production by geographic areas, institutional sectors, centers and thematic categories. The results were obtained after the analysis of 1302 documents, $75,19 \%$ of which are scientific articles. The most productive institutional sectors are hospitals $(54,8 \%)$ and universities $(43,9 \%)$. The most productive autonomous communities are Madrid $(28,2 \%)$ and Cataluña $(25,5 \%)$ as well as its various hospital and academic reference centers therein. The predominant categories end up being Clinical Neurology and Rehabilitation.
\end{abstract}

Keywords: Physiotherapy. Rehabilitation. Bibliometrics. Spain.

como de su situación en el contexto internacional (Bordons y Zulueta, 1999). Estos análisis se sirven de indicadores bibliométricos que proporcionan información cuantitativa y objetiva para valorar los resultados de la actividad científica.

La producción científica es un indicador que permite determinar el crecimiento de la ciencia a través del número de trabajos publicados y los diferentes elementos bibliográficos que contienen, de la producción de los investigadores, de un país o institución y las colaboraciones entre los mismos (Valera, 2001; Romaní, 2011). El presente trabajo pretende ofrecer una visión general de la producción científica desarrollada en España que permita contextualizar la Fisioterapia en este marco de estudio.

\section{Antecedentes}

El desarrollo de los estudios bibliométricos sobre Fisioterapia en España se centra en el análisis de la producción científica en las principales 
publicaciones nacionales de esta disciplina (Massó et al., 2000 y 2001; Martínez-González et al., 2002 y 2003; Martínez-Roldán et al., 2002; Valera, 2007; Meroño, 2010). García (2009) analiza las revistas que forman parte de los dos primeros cuartiles de los Journal Citation Reports 2007 para Rehabilitation. García et al. (2010) analizan los temas de investigación desarrollados internacionalmente en Fisioterapia según la Web of Science. Menéndez (2012) cuantifica cantidad y calidad de las noticias publicadas en la prensa escrita nacional desde el ámbito de fisioterapia. Martínez-Fuentes et al. (2013), identifican la base intelectual de la fisioterapia a través de análisis de cocitación. Calvo et al. (2013) determinan mediante análisis de co-palabras, los principales temas de investigación del dominio en España, como trabajo precedente al presente estudio.

\section{Material y métodos}

Este estudio utiliza como fuente de datos Science Citation Index $(\mathrm{SCl})$ y Social Science Citation Index (SSCl) de la Web of Science (WOS) producidas por el Institute for Scientific Information de Thomson Reuters.

Se realizó una búsqueda utilizando los términos Rehabilitation, Physical therapy y Physiotherapy en el campo topic, y Spain en el campo address, en el periodo comprendido desde los primeros documentos indexados en la base de datos hasta 2011. Se excluyeron del estudio aquellos documentos que no guardaran relación con el área de ciencias de la salud.

Se utilizaron indicadores bibliométricos relacionados con la tipología documental, la evolución temporal de la producción, el idioma de publicación; la distribución geográfica e institucional; los países con los que se colabora; así como la distribución temática en función de la adscripción de revistas.

\section{Resultados}

Se muestran los resultados obtenidos del análisis bibliométrico de los 1302 registros recuperados.

\subsection{Evolución temporal de la producción}

A pesar de que la cobertura temporal diseñada en la estrategia de búsqueda no parte de un año definido, el primer documento que se recupera data de 1972. No es hasta 1993 cuando la producción comienza a superar la decena de documentos. A partir del año 2000 la tendencia se muestra creciente de manera constante salvo excepciones en los años 2002, 2004 y 2005 (Gráfico1).

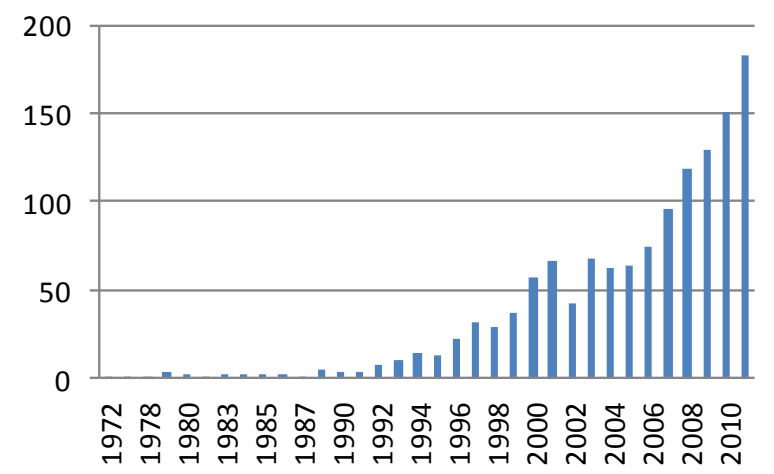

Gráfico 1. Evolución temporal de la producción

\subsection{Tipología documental}

Los principales tipos documentales son artículos científicos $(75,19 \%)$ y revisiones $(10,37 \%)$ seguidos de proceeding papers $(6,30 \%)$ y meeting abstracts (3,30\%) (Gráfico 2).

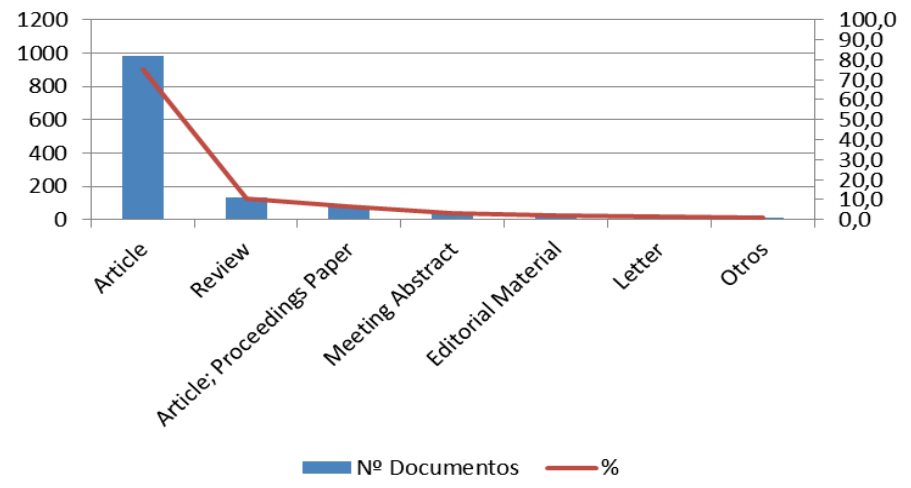

Gráfico 2. Tipología documental

\subsection{Idioma de publicación}

La mayor parte de la producción se publica en inglés $(75,88 \%)$ y en español $(15,67 \%)$, mientras que el $7,83 \%$ de los documentos no especifica el idioma (Gráfico 3).

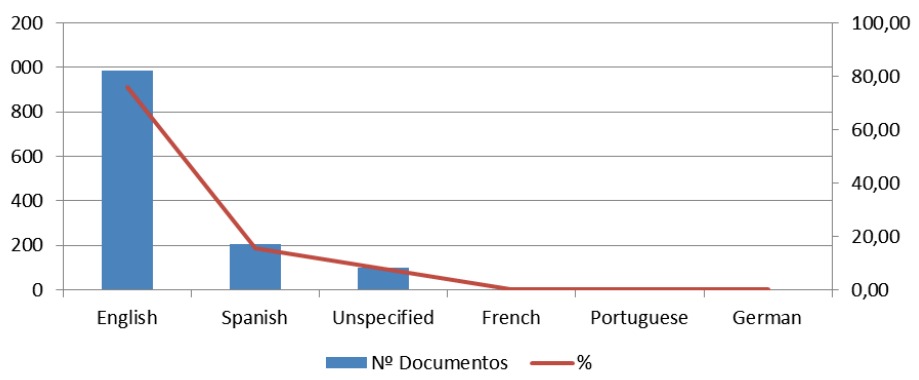

Gráfico 3. Idioma de publicación 


\subsection{Distribución por comunidades autónomas}

De las 17 comunidades autónomas españolas, las más productivas han sido Madrid, Cataluña, Andalucía y la Comunidad Valenciana. Destacan las dos primeras con más de la mitad $(53,7 \%)$ del total de documentos, seguidas de Andalucía y Comunidad Valenciana que contribuyen con el $12,1 \%$ y $11,9 \%$ de documentos respectivamente. Cabe destacar que estas cuatro Comunidades originan el $75 \%$ de las aportaciones totales y el resto se sitúan a cierta distancia, con porcentajes de producción bastante menores (Gráfico 4).

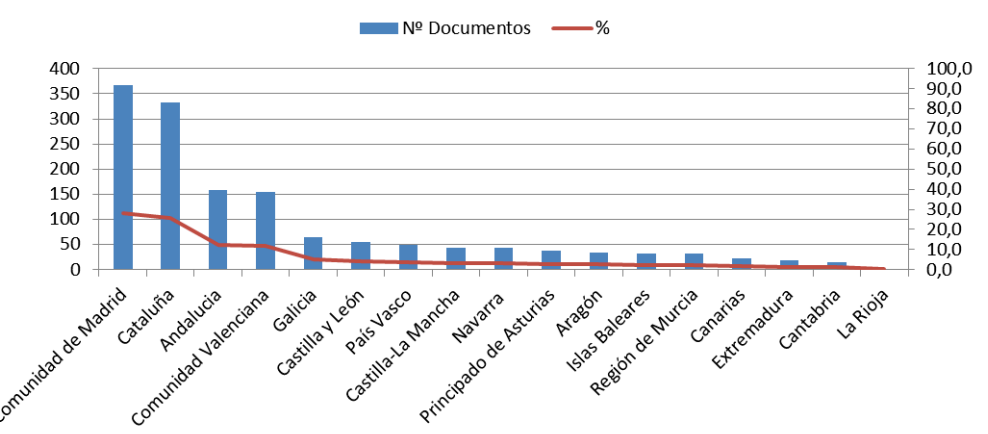

Gráfico 4. Distribución por comunidades autónomas

\subsection{Distribución por provincias}

Las provincias que han contribuido a la producción científica en el área de estudio han sido 48. Las que han participado en mayor medida guardan una estrecha relación con las comunidades autónomas más activas, destacando Madrid, Barcelona con el $52,4 \%$ de aportaciones, seguidas de Valencia con el 9,9\%. El resto contribuye con porcentajes de documentos poco significativos (Tabla I).

\begin{tabular}{lcr}
\hline Provincia & $N^{\circ}$ doc. & $\%$ \\
\hline Madrid & 368 & 28,3 \\
\hline Barcelona & 314 & 24,1 \\
\hline Valencia & 129 & 9,9 \\
\hline Sevilla & 57 & 4,4 \\
\hline Granada & 50 & 3,8 \\
\hline Coruña & 50 & 3,8 \\
\hline Navarra & 43 & 3,3 \\
\hline Asturias & 38 & 2,9 \\
\hline Málaga & 35 & 2,7 \\
\hline Vizcaya & 32 & 2,5 \\
\hline Islas Baleares & 32 & 2,5 \\
\hline Murcia & 32 & 2,5 \\
\hline Zaragoza & 31 & 2,4 \\
\hline Toledo & 28 & 2,2 \\
\hline
\end{tabular}

Tabla I. Provincias más productivas

\subsection{Distribución por sectores Institucionales}

El sector donde se desarrolla la mayor parte de la investigación en el área de estudio es el sanitario con un $54,8 \%$ de la producción total, seguido del sector universitario con un $43,9 \%$. Otros sectores activos son los dependientes de la Administración Autonómica $(8,8 \%)$, y los Organismos públicos de investigación (Consejo Superior de Investigaciones Científicas e Instituto de Salud Carlos III) con el 6,7\% (Gráfico 5).
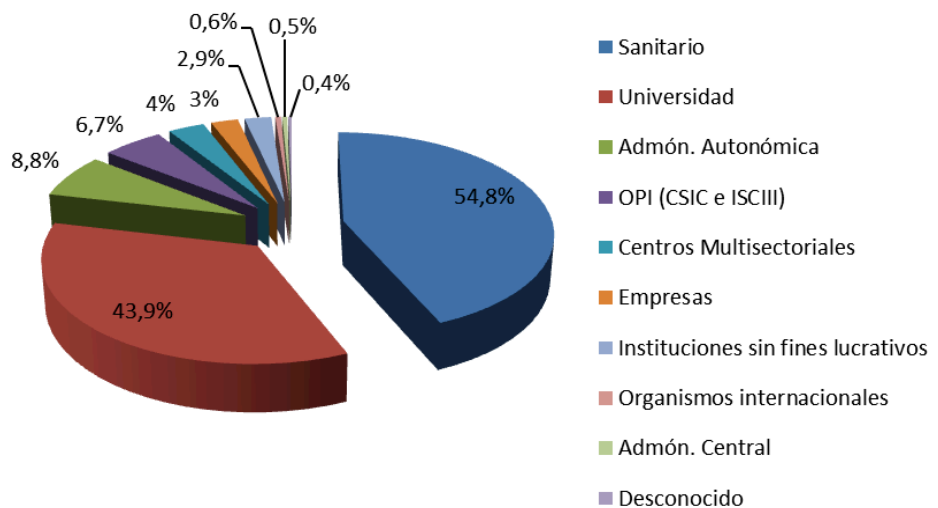

Gráfico 5. Distribución por Sectores Institucionales

\subsection{Distribución por Centros}

La producción documental se distribuye en 546 Centros, de los que tan solo 22 contribuyen con más de 20 documentos, lo que supone más de la mitad de la producción $(53,46 \%)$. Destacan la Universidad Complutense de Madrid, el Hospital Universitario de La Paz con más de 50 aportaciones cada uno (se han considerado de forma independiente los documentos procedentes de centros sanitarios adscritos a universidades); seguidos de la Universidad de Valencia, la Universidad de Granada y el Instituto Guttmann, adscrito a la Universidad Autónoma de Barcelona, que superan las 40 contribuciones (Tabla II).

\begin{tabular}{lcc}
\hline Centro & $N^{\circ}$ doc. & $\%$ \\
\hline Univ Complutense de Madrid (Madrid) & 54 & 4,15 \\
\hline Hosp Univ de La Paz (Madrid) & 52 & 3,99 \\
\hline Univ Valencia (Valencia) & 49 & 3,76 \\
\hline Univ Granada (Granada) & 43 & 3,30 \\
\hline Inst Guttmann (Barcelona) & 40 & 3,07 \\
\hline Univ Rey Juan Carlos (Madrid) & 36 & 2,76 \\
\hline Hosp Sta Cruz y San Pablo (Barcelona) & 34 & 2,61 \\
\hline Univ Barcelona (Barcelona) & 33 & 2,53 \\
\hline Hosp Univ y Politécnico de La Fe de Valencia & 31 & 2,38 \\
\hline Hosp Gral Valle Hebrón (Barcelona) & 30 & 2,30 \\
\hline Hosp Gregorio Marañón (Madrid) & 29 & 2,23 \\
\hline Hosp Clin Barcelona (Barcelona) & 29 & 2,23 \\
\hline Univ Autónoma de Barcelona (Barcelona) & 26 & 2,00 \\
\hline Hosp Univ Virgen del Rocio (Sevilla) & 26 & 2,00 \\
\hline
\end{tabular}




\begin{tabular}{lll}
\hline Inst Invest Biomed August Pi i Sunyer (Barcelona) & 26 & 2,00 \\
\hline Hosp Ramón y Cajal (Madrid) & 26 & 2,00 \\
\hline Univ Sevilla (Sevilla) & 25 & 1,92 \\
\hline Univ Murcia (Murcia) & 24 & 1,84 \\
\hline Univ Santiago de Compostela (La Coruña) & 21 & 1,61 \\
\hline Hosp 12 Octubre (Madrid) & 21 & 1,61 \\
\hline Univ Oviedo (Oviedo) & 21 & 1,61 \\
\hline Parque de Salud Mar (Barcelona) & 20 & 1,54
\end{tabular}

Tabla II. Centros más productivos

\subsection{Colaboración internacional}

La creación de equipos de investigación de varios países resulta favorable, ya que contribuye al aumento de la visibilidad de los trabajos. Las colaboraciones internacionales en este estudio suponen el $35 \%$ del total de documentos. Destaca Estados Unidos como principal colaborador (14,1\%), seguido de países como Italia, Inglaterra y Alemania. En el Gráfico 6 se muestran los países que han participado como socios colaboradores con más de 20 documentos.

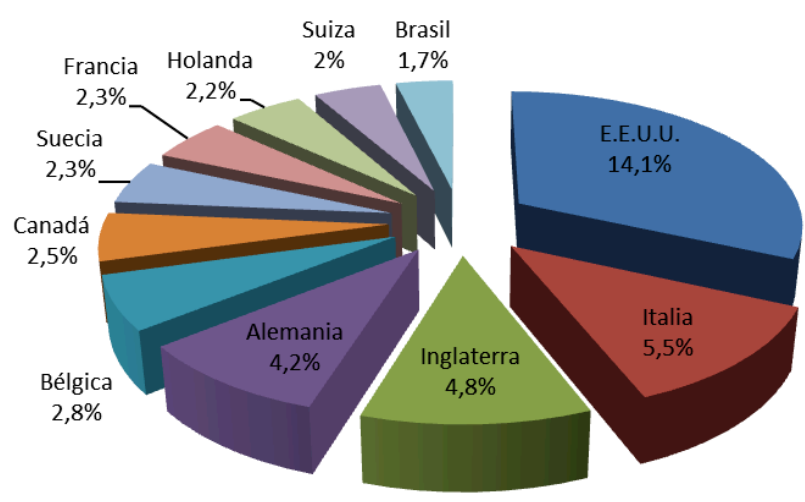

Gráfico 6. Colaboraciones más frecuentes

\subsection{Revistas de publicación}

El total de revistas en las que se publicaron los documentos es de 505, lo que sugiere una notable dispersión del dominio estudiado. Destaca la productividad de la Revista de Neurología (107 documentos) y Archives of Physical Medicine and Rehabilitation (72 documentos) que agrupan el $13,7 \%$ del total, seguidas de varias publicaciones situadas a gran distancia en relación al volumen de producción (Tabla III).

\subsection{Análisis de categorías}

Los documentos se publicaron en revistas clasificadas en 134 categorías temáticas. Destacan especialmente Clinical Neurology y Rehabilitation que aglutinan el $37,8 \%$ del total de documentos. Junto a ellas destacan también Sport Sciences y Neurosciences con 148 y 113 documentos respectivamente (Tabla IV).

\begin{tabular}{lrc}
\hline Revista & $N^{\circ}$. doc. & $\%$ \\
\hline Revista de Neurología & 107 & 8,2 \\
\hline Archives of Physical Medicine and Rehabilitation & 72 & 5,5 \\
\hline Archivos de Bronconeumología & 30 & 2,3 \\
\hline Medicina Clínica & 29 & 2,2 \\
\hline Haemophilia & 20 & 1,5 \\
\hline Revista Española de Cardiología & 20 & 1,5 \\
\hline Actas Españolas de Psiquiatría & 19 & 1,5 \\
\hline Medicina Oral Patología Oral y Cirugía Bucal & 18 & 1,4 \\
\hline Neurología & 18 & 1,4 \\
\hline Brain Injury & 17 & 1,3 \\
\hline American Journal of Physical Medicine \& Rehab. & 16 & 1,2 \\
\hline
\end{tabular}

Tabla III. Revistas más productivas

\begin{tabular}{lrr}
\hline Categoria & $N^{\circ}$ doc. & $\%$ \\
\hline Clinical Neurology & 259 & 19,9 \\
\hline Rehabilitation & 233 & 17,9 \\
\hline Sport Sciences & 148 & 11,4 \\
\hline Neurosciences & 113 & 8,7 \\
\hline Dentistry, Oral Surgery \& Medicine & 77 & 5,9 \\
\hline Respiratory System & 77 & 5,9 \\
\hline Orthopedics & 72 & 5,5 \\
\hline Medicine, General \& Internal & 68 & 5,2 \\
\hline Surgery & 67 & 5,1 \\
\hline Psychiatry & 57 & 4,4 \\
\hline Cardiac \& Cardiovascular Systems & 43 & 3,3 \\
\hline Health Care Sciences \& Services & 36 & 2,8 \\
\hline Engineering, Biomedical & 32 & 2,5 \\
\hline
\end{tabular}

Tabla IV. Categorías temáticas más frecuentes

\section{Discusión y conclusiones}

La producción total española recogida en el SCI y SSCl hasta 2011 se concentra en 32 años, aunque es en los últimos 10 años cuando experimenta un mayor crecimiento. Se presenta mayoritariamente en formato de artículo científico en lengua inglesa.

Predominan las contribuciones del sector sanitario, aunque dentro de las instituciones más productivas se encuentran el mismo número de hospitales que de universidades. La mayor producción se sitúa en la Comunidad de Madrid seguida de Cataluña y Andalucía, y se concentra en varios centros hospitalarios y universitarios de referencia de las mismas.

Se aprecia una considerable colaboración investigadora entre diversos países, lo que favorece mayor difusión y visibilidad internacional, encabezada en este caso por Estados Unidos.

En líneas generales, los datos obtenidos en el presente estudio reproducen las tendencias de la producción biomédica española descritas por Camí et al. (2007) y Méndez-Vásquez et al. (2008), por lo que se puede afirmar que esta disciplina sigue los patrones que caracterizan la producción española en biomedicina. 
Por otro lado, cabe señalar que la investigación española en Rehabilitación y Fisioterapia aún es discreta. Aunque, si bien es cierto que la base de datos WOS sólo muestra la investigación más visible a nivel internacional, consideramos que en aras de una mayor relevancia, se debería fomentar el uso de revistas incluidas en esta base de datos.

La importante transversalidad de esta disciplina, que se refleja por el elevado número de categorías temáticas, hace necesario buscar métodos alternativos que reflejen con mayor especificidad líneas de investigación especializadas, como pueda ser el análisis de redes, que se identifica como una clara línea de trabajo futuro.

\section{Referencias}

Bordons, M.; Zulueta-García, M. A. (1999). Evaluación de la actividad científica a través de indicadores bibliométricos. // Revista Española de Cardiología. 52:10 (1999) 790-800.

Camí, J.; Suñén-Piñol, E.; Méndez-Vásquez, R. I. (2005). Mapa bibliométrico de España 1994-2002: biomedicina y ciencias de la salud. // Medicina Clínica. 124 (2005) 93101.

Calvo-Fuente, V.; Cantos-Mateos, G.; Zulueta-García, M. A. (2013). Delimitación temática de la investigación española en Fisioterapia a través del análisis de co-palabras. // Scire. 19:2 (2013) 98-101.

García-Ríos, M. C. (2009). El paradigma de la Fisioterapia a través de un estudio ciencimétrico. Universidad de Granada. Tesis Doctoral.

García-Ríos, M. C.; Moreno-Lorenzo, C.; Ruiz- Baños, R.; Bailón-Moreno, R. (2010). Análisis temático de la disciplina Fisioterapia en la Web of Science. // Fisioterapia. 32:4 (1999) 159-64.

Martínez-González, M.; Gómez-Conesa, A. (2003). Estudio bibliométrico de la Revista Iberoamericana de Fisioterapia y Kinesiología (1998-2002). // Revista Iberoamericana de Fisioterapia y Kinesiología. 6:1 (2003) 58-71.
Martínez-González, M.; Marín-Fernández, J.; RebolloRoldán, J. (2002). Producción científica de la revista Cuestiones de Fisioterapia (1995-2000). // Cuestiones de Fisioterapia. 20 (2002) 1-11.

Massó-Ávila, J. J.; Bernabéu-Lledó, M.; Medina-Mirapeix, F. (2000). Productividad de los fisioterapeutas españoles en el período 1991-1999 a través del análisis bibliométrico de la revista Fisioterapia. // Fisioterapia. 22:1 (2000) 2-11.

Massó-Avila, J. J.; Valera-Garrido, J. F.; Medina-Mirapeix, F.; M. (2001). Análisis del consumo de información en la revista Fisioterapia (1991-1999). // Fisioterapia. 23:3 (2001) 154-159.

Méndez-Vásquez, R. I.; Suñén-Pinyol, E.; Cervelló R.; Camí, J. (2008). Mapa bibliométrico de España 1996-2004: biomedicina y ciencias de la salud // Medicina Clínica. 130:7 (2008) 246-53.

Menéndez, R. (2012). Avances de la fisioterapia en la prensa escrita. Cuantificación de la transferencia de conocimiento científico desde el ámbito de la fisioterapia hacia la sociedad. // Fisioterapia. 34:4 (2012) 161-168.

Meroño-Gallut, A. J. (2010). Desarrollo científico de la Fisioterapia en España. Estudio de los artículos publicados en la Revista Fisioterapia (1979-2006). Universidad de Sevilla. Tesis Doctoral.

Romaní, F.; Huamaní, C.; González-Alcaide, G. (2011). Estudios bibliométricos como línea de investigación en las ciencias biomédicas: una aproximación para el pregrado. // CIMEL 16:1 (2011) 52-62.

Torres Narváez, M., Hernández Jaramillo, J.; Cruz Velandia, I. (2012). Análisis de la producción de literatura científica en las áreas de investigación clínica en Fisioterapia entre los años 2005 y 2009. // Revista Ciencias de la Salud. 10:1 (2012) 33-42.

Valera-Garrido, JF. (2007). La investigación en Fisioterapia en España a través del análisis bibliométrico de la Revista Fisioterapia (1991-1999). Universidad de Murcia. Tesis Doctoral.

Valera-Garrido J. F.; de la Gala-Sánchez, F. (2001). Análisis bibliométrico de la productividad científica en la revista MAPFRE Medicina. // MAPFRE Medicina. 12:3 (2001) 717.

Enviado: 2014-04-04. Aceptado: 2014-04-04. 
